\title{
LOCAL NON-SIMILARITY SOLUTIONS FOR A FORCED-FREE BOUNDARY LAYER FLOW WITH VISCOUS DISSIPATION
}

\author{
E.W. Mureithi ${ }^{1}$ and D.P. Mason ${ }^{2}$ \\ ${ }^{1}$ Department of Mathematics and Applied Mathematics, \\ University of Pretoria, South Africa. \\ 2 School of Computational and Applied Mathematics, \\ University of the Witwatersrand, South Africa. \\ eunice.mureithi@up.ac.za, david.mason@wits.ac.za
}

\begin{abstract}
The boundary layer flow over a horizontal plate with power law variations in the freestream velocity and wall temperature of the form $U_{e} \sim x^{n}$ and $T_{w}-T_{\infty} \sim x^{m}$ and with viscous dissipation, is studied. The boundary layer equations are transformed to a dimensionless system of equations using a non-similarity variable $\xi(x)$ and a pseudo-similarity variable $\eta(x, y)$. The effects of the various parameters of the flow on velocity and temperature distribution in the boundary layer, on the local skin friction and local heat transfer coefficients and on the non-similar terms, are investigated.
\end{abstract}

Keyword- Boundary layer; Non-similarity; Viscous dissipation; Buoyancy; Temperature overshoot; Super-velocities.

\section{INTRODUCTION}

Studies on forced-free boundary layers have been on the increase due to their wide range of applications in engineering and in industry.

Prandtl's boundary layer equations where the free-stream velocity varies as $U_{e}(x) \propto x^{n}$ normally admit similarity type solutions. Non-similarity of boundary layers may arise from such factors as variation in free-stream velocity, effect of suction or injection of fluid at the wall, variation in wall temperature and the buoyancy force effect. In some cases non-similarity is caused by more than one factor.

For a forced-free convection boundary layer, non-similarity of the flow field leads to a non-similar thermal field. Cases may arise where the velocity boundary layer is similar but the thermal boundary layer is non-similar. Extensive studies have been done for mixed convection boundary layer flows. These include the early work of Mori [3] and Sparrow and Minkowycz [9] who studied the mixed convection boundary layer flow over a flat plate; the work of Sparrow and Yu [10] who 
described and applied the local non-similar method to solve the thermal boundary layer equations; and the work of Ramachandran et al [6] who studied mixed convection over a heated horizontal plate for an entire range of the buoyancy parameter. Later work by Raju et al [5] analyzed the problem considered by Ramachandran et $a l$ [6] by using a mixed convection parameter ranging from 0 (pure free convection) to 1 (pure forced convection). Risbeck [7] used a single valued mixed convection parameter to re-examine the mixed convection problem for power-law variation in the wall temperature. Recent work by Hassanien et al [2] used the local non-similar method to analyse a mixed convection boundary layer flow of a micropolar fluid over a horizontal plate, where a nonsimilar mixed convection parameter is used to define the entire convection range.

The present research was motivated by a recent study by Mureithi and Mason [4] on the stability of a mixed convection boundary layer with viscous heating where it was shown that this boundary layer flow is generally non-similar except for the one special case of stagnation point flow. The flow becomes non-similar due to the combined effects of thermal buoyancy and the viscous dissipation. Rogers [12] has extensively used the local non-similarity method in analysing various types of non-similar boundary layers. The work includes a study of a forced-free convection boundary layer flow over a vertical wall, with thermal buoyancy, viscous dissipation and suction or injection. The results presented by Rogers [12] include the effects of thermal buoyancy on the velocity and temperature distributions in the boundary layer. In the current study we look at a flow over a horizontal wall. We focus on the effects that thermal buoyancy, viscous dissipation and the free-stream exponent $n$, have on the skin friction, heat transfer, velocity and temperature distribution and on the non-similar terms in the momentum and the thermal boundary layers.

There are various numerical techniques for solving local non-similar boundary layer problems. In the work presented here we apply the local non-similar method developed by Sparrow and co-workers ([11], [10]). The method involves transforming the physical coordinates $(x, y)$ to appropriately selected dimensionless coordinates $(\xi, \eta), \eta$ being the pseudo-similarity variable and $\xi$ the non-similarity variable. When $\xi=0$ or $\xi=$ constant, the boundary layer flow becomes similar. Rogers [12] has presented results comparing the local non-similar method with the finite difference method, for the solution of a two-dimensional boundary layer with pressure gradient. The results agree very well at the second or third level of truncation. Some of the advantages of using this method are the fact that it is simple to implement, the partial differential equations are reduced to ordinary differential equations and each solution is locally independent of the solution at a previous streamwise location. 


\section{THE STEADY BOUNDARY LAYER EQUATIONS.}

Consider the steady boundary layer flow of a viscous incompressible fluid, of density $\rho$ and kinematic viscosity $\nu$, over a heated semi-infinite flat plate. The horizontal plate coincides with the $x$-axis and the $y$-axis is in the normal direction to the flow. The dimensional boundary layer equations, under the boundary layer approximation and the Boussinesq approximation, are

$$
\left.\begin{array}{rl}
u \frac{\partial u}{\partial x}+v \frac{\partial u}{\partial y} & =-\frac{1}{\rho} \frac{\partial p}{\partial x}+\nu \frac{\partial^{2} u}{\partial y^{2}}, \\
\frac{1}{\rho} \frac{\partial p}{\partial y} & =g \alpha\left(T-T_{\infty}\right), \\
u \frac{\partial T}{\partial x}+v \frac{\partial T}{\partial y} & =\frac{\kappa}{\rho c_{p}} \frac{\partial^{2} T}{\partial y^{2}}+\frac{\nu}{c_{p}}\left(\frac{\partial u}{\partial y}\right)^{2},
\end{array}\right\}
$$

where $g$ is the acceleration due to gravity, $T_{\infty}(x)$ is the free-stream temperature, $c_{p}$ is the coefficient of specific heat of the fluid at constant pressure and $\alpha$ and $\kappa$ are the coefficients of volume expansion and thermal conductivity, respectively.

The free-stream velocity and the plate temperature are assumed to vary in the stream-wise direction. Hence we have the boundary conditions

$$
\left.\begin{array}{l}
u(x, 0)=v(x, 0)=0, \quad T(x, 0)=T_{w}(x), \\
u(x, \infty)=U_{e}(x), \quad T(x, \infty)=T_{\infty},
\end{array}\right\}
$$

where $T_{w}(x)$ is the wall temperature and $U_{e}(x)$ the velocity at the edge of the boundary layer.

\section{NON-SIMILAR BOUNDARY LAYER EQUATIONS}

We make the following transformations (see Sparrow and Minkowycz [9]) in the boundary layer equations with thermal buoyancy

$$
\begin{aligned}
& \xi=\frac{G r_{x}}{R e_{x}^{5 / 2}}, \quad \eta=\frac{y}{x} R e_{x}^{1 / 2}, \quad u(x, y)=U_{e} f^{\prime}(\xi, \eta), \\
& T-T_{\infty}=\left(T_{w}-T_{\infty}\right) h(\xi, \eta), \quad p(x, y)=\rho U_{e}^{2}\left(q(\xi, \eta)-\frac{1}{2}\right),
\end{aligned}
$$

where the prime denotes differentiation with respect to $\eta$. Here $\xi(x)$ is a nonsimilarity variable and $\eta(x, y)$ is a boundary layer pseudo-similarity variable. The dimensionless quantities $R e_{x}$ and $G r_{x}$ are the local Reynolds number and the local Grashof number defined as

$$
R e_{x}=\frac{U_{e} x}{\nu}, \quad G r_{x}=\frac{g \alpha\left(T_{w}-T_{\infty}\right) x^{3}}{\nu^{2}}
$$


From the equation of continuity, it follows that

$$
v(x, y)=-\frac{U_{e}}{2 R e_{x}^{1 / 2}}\left[(\beta+1) f+\xi \gamma \frac{\partial f}{\partial \xi}+\eta(\beta-1) f^{\prime}\right],
$$

where

$$
\beta(\xi)=\frac{x}{U_{e}} \frac{d U_{e}}{d x}, \quad \sigma(\xi)=\frac{x}{T_{w}-T_{\infty}} \frac{d T_{w}}{d x}, \quad \gamma=1-5 \beta+2 \sigma .
$$

Substitution of these variables into the boundary layer equations (1) gives the nonsimilar boundary layer equations

$$
\begin{aligned}
f^{\prime \prime \prime}= & \beta\left[\left(f^{\prime}\right)^{2}-1+2 q\right]-\frac{1}{2}(\beta+1) f f^{\prime \prime}+\frac{1}{2}(\beta-1) \eta q^{\prime} \\
& +\frac{1}{2} \xi \gamma\left[f^{\prime} \frac{\partial f^{\prime}}{\partial \xi}-f^{\prime \prime} \frac{\partial f}{\partial \xi}+\frac{\partial q}{\partial \xi}\right], \\
q^{\prime}= & \xi h, \\
h^{\prime \prime}= & \frac{1}{2} \operatorname{Pr}\left\{2 \sigma h f^{\prime}-(\beta+1) f h^{\prime}-2 E c\left(f^{\prime \prime}\right)^{2}\right\} \\
& +\frac{1}{2} \operatorname{Pr} \xi \gamma\left[f^{\prime} \frac{\partial h}{\partial \xi}-h^{\prime} \frac{\partial f}{\partial \xi}\right] .
\end{aligned}
$$

The appropriate boundary conditions are

$$
\left.\begin{array}{l}
f^{\prime}(\xi, 0)=0, \quad(1+\beta) f(\xi, 0)+\xi \gamma \frac{\partial f}{\partial \xi}(\xi, 0)=0, \quad h(\xi, 0)=1, \\
f^{\prime}(\xi, \infty)=1, \quad h(\xi, \infty)=0, \quad q(\xi, \infty)=0 .
\end{array}\right\}
$$

The parameters $E c$ and $\operatorname{Pr}$ are the local Eckert number and the Prandtl number defined as

$$
E c=\frac{U_{e}^{2}}{c_{p}\left(T_{w}-T_{\infty}\right)}, \quad \operatorname{Pr}=\frac{\mu c_{p}}{\kappa} .
$$

The flow considered here is boundary layer flow of a fluid of Prandtl number $\operatorname{Pr}=0.7$ (air). The temperature difference between the wall and the free-stream is approximately $10 \mathrm{~K}$ and $c_{p} \approx 10^{3}$. Hence the Eckert number is of order one if $U_{e} \approx 10^{2}$.

The wall shear stress can be represented using the local skin friction coefficient $C_{f}$, and the heat transfer coefficient is measured using the local Nusselt number, $N u_{x}$, defined respectively as

$$
\frac{1}{2} R e_{x}^{1 / 2} C_{f}=f^{\prime \prime}(\xi, 0), \quad R e_{x}^{-1 / 2} N u_{x}=-h^{\prime}(\xi, 0) .
$$


Assuming power law variations in the free-stream velocity and in the plate temperature of the form

$$
U_{e}(x) \propto x^{n}, \quad T_{w}-T_{\infty} \propto x^{m},
$$

gives that $\beta=n$ and $\sigma=m$. For steady inviscid flow past a wedge $n \leq 1$. We therefore restrict ourselves to $n \leq 1$. The case when $n=1$ is the self-similar solution derived by Mureithi and Mason [4].

In solving (3) to (5) we follow closely the local non-similar method developed by Sparrow and $\mathrm{Yu}[10]$. The equations are derived to a third level of truncation.

For convenience, a subscript 1 and 2 denote differentiation of the original nonsimilar equations, once and twice, with respect to $\xi$; hence for example,

$$
\begin{gathered}
f_{1}=\frac{\partial f}{\partial \xi}, \quad f_{1}^{\prime}=\frac{\partial f^{\prime}}{\partial \xi}, \theta_{1}=\frac{\partial \theta}{\partial \xi}, \cdots \\
f_{2}=\frac{\partial f_{1}}{\partial \xi}, \quad f_{2}^{\prime}=\frac{\partial f_{1}^{\prime}}{\partial \xi}, \quad \theta_{2}=\frac{\partial \theta_{1}}{\partial \xi}, \cdots
\end{gathered}
$$

The equations (3), (4) and (5) to second and third level of approximation are

$$
\begin{aligned}
f_{1}^{\prime \prime \prime}= & \frac{1}{2}(3 n+1)\left[f_{1}^{\prime} f^{\prime}+q_{1}\right]-f_{1} f^{\prime \prime}-\frac{1}{2}(n+1) f f_{1}^{\prime \prime} \\
& +\frac{1}{2}(n-1) \eta q_{1}^{\prime}+\frac{1}{2}(1-n) \xi\left[\left(f_{1}^{\prime}\right)^{2}-f_{1}^{\prime \prime} f_{1}\right], \\
q_{1}^{\prime}= & h+\xi h_{1}, \\
h_{1}^{\prime \prime}= & \frac{1}{2} \operatorname{Pr}\left\{(3 n+1) h_{1} f^{\prime}+4 n h f_{1}^{\prime}-2 f_{1} h^{\prime}-(1+n) f h_{1}^{\prime}\right. \\
& \left.-4 E c f^{\prime \prime} f_{1}^{\prime \prime}+(1-n) \xi\left[f_{1}^{\prime} h_{1}-h_{1}^{\prime} f_{1}\right]\right\},
\end{aligned}
$$

with boundary conditions, valid for all values of $n$,

$$
\left.\begin{array}{l}
f_{1}(\xi, 0)=0, \quad f_{1}^{\prime}(\xi, 0)=0, \quad h_{1}(\xi, 0)=0, \\
f_{1}^{\prime}(\xi, \infty)=0, \quad h_{1}(\xi, \infty)=0, \quad q_{1}(\xi, \infty)=0 .
\end{array}\right\}
$$

The solution of this system of equations and boundary conditions is presented in the next section.

The method of Sparrow and $\mathrm{Yu}[10]$ is discussed in greater detail in the text book by Minkowycz et al [1]. 


\section{NUMERICAL SOLUTIONS}

The non-similarity solutions are obtained by solving together the boundary layer equations (3) to (6) and the auxiliary system (7) to (10). These equations can be rewritten as a system of twelve first order partial differential equations with twelve boundary conditions for twelve unknowns. At any position $\xi(x)$, the twelve first order equations are solved as if they were ordinary differential equations with $\xi$ being treated as a buoyancy parameter. The system is solved using the fourth-order Runge-Kutta integrator and a Newton-Raphson iterative scheme.

\section{DISCUSSION OF RESULTS}

The results presented here are for $\operatorname{Pr}=0.7$, except for Figures 1 and 2 where the effect of varying the Prandtl number is investigated. Increasing the buoyancy parameter $\xi$ is equivalent to increasing buoyancy forces.

Figures 1 and 2 show the variation of $f^{\prime \prime}(\xi, 0)$ and $-h^{\prime}(\xi, 0)$ with $\xi$, respectively. Increase in the buoyancy parameter, $\xi$, results in increase in the skin friction coefficient and decrease in the surface heat transfer rate. The skin friction factor is higher in fluids of low Prandtl number for the case when $\xi>0$, the reverse occurring when $\xi<0$. The surface heat transfer also increases with decrease in Prandtl number. When $\xi=0$, there is no coupling between the temperature and the velocity fields and hence varying the Prandtl number has no effect on the skin friction factor, as seen in Figure 1.

Figures 3 to 6 show the effect of increasing $\xi$ and $E c$ for some fixed value of $n$ chosen to be $n=0.2$. The parameter $E c$ is a measure of the viscous dissipation effects. These figures show that increasing $E c$ (for $\xi=1.5$ ) and increasing $\xi$ (for $E c=1)$ results in a decrease in the thickness of both the momentum and the thermal boundary layers.

The results indicate that velocity "overshoots" occur inside the boundary layer when $n=0.2, E c=1$ and for $\xi \geq 0.09$. Temperature "overshoots" also are observed to occur near the wall when $n=0.2, E c=1$ and when $\xi \geq 1.2$. By "overshoot" we mean that the values for the velocity and temperature exceed the free-stream value and the plate temperature, respectively. The "super-velocities" and temperature "overshoots" are due to the combined effect of buoyancy and viscous dissipation. These results are in total agreement with those obtained by Mureithi and Mason [4] for the case when $n=1$.

The effect of varying $n$ on $f^{\prime \prime}(\xi, 0)$ and $-h^{\prime}(\xi, 0)$ is shown in Figures 7 and 8, respectively. For $n<0$ there is a critical value of $n$ dependent on $\xi$ beyond which there is no solution. For $\xi=0$ the critical value of $n$ obtained here is $n_{c r} \approx-0.09$ 
which is consistent with the results for a non-thermal boundary layer described by the Faulkner-Skan equation. For $n<-0.0904$, there are no normal solutions with $0<f^{\prime}(\eta)<1$ of the Faulkner-Skan equation and only solutions with reversed flow may exist ( Schlichting [8]) For $\xi=0$ (no buoyancy force), there is a small interval where the solution is not unique. When buoyancy is taken into account $(\xi \neq 0)$, unique solutions exist for all $n \geq n_{c r}$ and $n_{c r}$ increases with increase of $\xi$. The skin friction decreases with decrease in $n$ but starts to rise quite sharply as $n \rightarrow n_{c r}$. The heat transfer coefficient decreases monotonically with decrease in $n$, with the decrease becoming more sharp as $n \rightarrow n_{c r}$.

The effect of varying $n$ on $f^{\prime}(\xi, \eta)$ and $h(\xi, \eta)$ is shown in Figures 9 and 10. The magnitude of the velocity and temperature overshoots increases with decrease in $n$. A decrease in $n$ translates to an increase in favourable pressure gradient while an increase in $n$ means that the flow is a decelerating flow due the effect of an adverse pressure gradient.

Figures 11 to 14 show the variation of the non-similar terms with $\xi$ and $n$. The non-similarity effects increase significantly with increase in $\xi$ and decrease in $n$. The results indicate that the non-similarity effects are more pronounced in the velocity boundary layer than in the thermal boundary layer.

\section{CONCLUSION}

A non-similarity model has been presented for a buoyant boundary layer flow with viscous dissipation. This model reduces to the similarity model when $n=1$. Solutions obtained for the case $n=1$ agree with the results of Mureithi and Mason [4]. Using the non-similarity model we have obtained solutions for cases when $n \leq 1$.

Super-velocities have been observed and their magnitude increases with increase in both $\xi$ and $E c$ and with decrease in $n$. The velocity overshoots occur in a region inside the boundary layer. These overshoots result because at high values of $\xi$ the temperature induced horizontal pressure gradient plays a role similar to that of a favourable pressure gradient in accelerating the flow in boundary layer. Temperature overshoots (also referred to as Sparrow-Greg type "hills") have also been observed near the plate since viscous dissipation effects are strongest there.

Our results indicate that for a fixed value of $\xi$, there is a critical value of $n$ below which there are no solutions. This value increases with increase in $\xi$.

The variation of the non-similar terms in the velocity and the thermal boundary layer has also been studied. These terms become significant as $n$ decreases and as $\xi$ increases. The non-similarity effects are stronger in the velocity boundary layer 
than the thermal boundary layer.

Acknowledgement- This material is based upon research supported by the National Research Foundation, Pretoria, South Africa under Grant number 2053745. DPM and EWM thank the NRF for its financial support. EWM was also supported by Research and Development fund research fund, University of Pretoria. EWM thanks the University of Pretoria for its financial support.

The results shown are for the case when $E c=1, n=0.2$ and $\operatorname{Pr}=0.7$.

\begin{tabular}{|clc|}
\hline \hline$\xi$ & $f^{\prime \prime}(\xi, 0)$ & $-h^{\prime}(\xi, 0)$ \\
\hline$-0.7000 \mathrm{E}-01$ & $0.3725 \mathrm{E}+00$ & $0.1755 \mathrm{E}+00$ \\
$-0.5000 \mathrm{E}-01$ & $0.4525 \mathrm{E}+00$ & $0.1799 \mathrm{E}+00$ \\
$-0.1000 \mathrm{E}-01$ & $0.5903 \mathrm{E}+00$ & $0.1840 \mathrm{E}+00$ \\
$0.5550 \mathrm{E}-17$ & $0.6213 \mathrm{E}+00$ & $0.1844 \mathrm{E}+00$ \\
$0.2000 \mathrm{E}+00$ & $0.1118 \mathrm{E}+01$ & $0.1781 \mathrm{E}+00$ \\
$0.4000 \mathrm{E}+00$ & $0.1511 \mathrm{E}+01$ & $0.1585 \mathrm{E}+00$ \\
$0.6000 \mathrm{E}+00$ & $0.1859 \mathrm{E}+01$ & $0.1327 \mathrm{E}+00$ \\
$0.8000 \mathrm{E}+00$ & $0.2180 \mathrm{E}+01$ & $0.1027 \mathrm{E}+00$ \\
$0.1000 \mathrm{E}+01$ & $0.2484 \mathrm{E}+01$ & $0.6932 \mathrm{E}-01$ \\
$0.1200 \mathrm{E}+01$ & $0.2775 \mathrm{E}+01$ & $0.3310 \mathrm{E}-01$ \\
$0.1400 \mathrm{E}+01$ & $0.3057 \mathrm{E}+01$ & $-0.5731 \mathrm{E}-02$ \\
\hline
\end{tabular}

\begin{tabular}{|clc|}
\hline \hline$n$ & $f^{\prime \prime}(\xi, 0)$ & $-h^{\prime}(\xi, 0)$ \\
\hline$-0.1800 \mathrm{E}+00$ & $0.2144 \mathrm{E}+01$ & $-0.1955 \mathrm{E}+01$ \\
$-0.1000 \mathrm{E}+00$ & $0.1502 \mathrm{E}+01$ & $-0.5572 \mathrm{E}+00$ \\
$-0.2000 \mathrm{E}-01$ & $0.1399 \mathrm{E}+01$ & $-0.2451 \mathrm{E}+00$ \\
$0.0000 \mathrm{E}+00$ & $0.1394 \mathrm{E}+01$ & $-0.2008 \mathrm{E}+00$ \\
$0.8000 \mathrm{E}-01$ & $0.1405 \mathrm{E}+01$ & $-0.7693 \mathrm{E}-01$ \\
$0.1200 \mathrm{E}+00$ & $0.1421 \mathrm{E}+01$ & $-0.3342 \mathrm{E}-01$ \\
$0.2000 \mathrm{E}+00$ & $0.1462 \mathrm{E}+01$ & $0.3450 \mathrm{E}-01$ \\
$0.2800 \mathrm{E}+00$ & $0.1507 \mathrm{E}+01$ & $0.8710 \mathrm{E}-01$ \\
$0.3600 \mathrm{E}+00$ & $0.1555 \mathrm{E}+01$ & $0.1305 \mathrm{E}+00$ \\
$0.4400 \mathrm{E}+00$ & $0.1602 \mathrm{E}+01$ & $0.1680 \mathrm{E}+00$ \\
$0.5000 \mathrm{E}+00$ & $0.1637 \mathrm{E}+01$ & $0.1931 \mathrm{E}+00$ \\
\hline
\end{tabular}




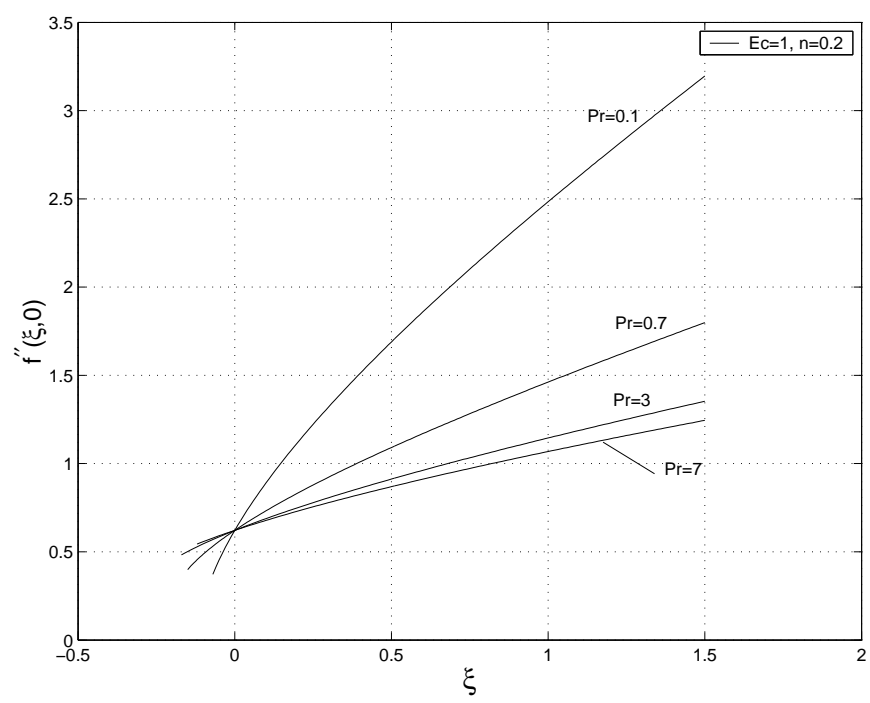

Figure 1: $f^{\prime \prime}(\xi, 0)$ plotted against $\xi$, for $E c=1, n=0.2$ and $\operatorname{Pr}=0.1,0.7,7$.

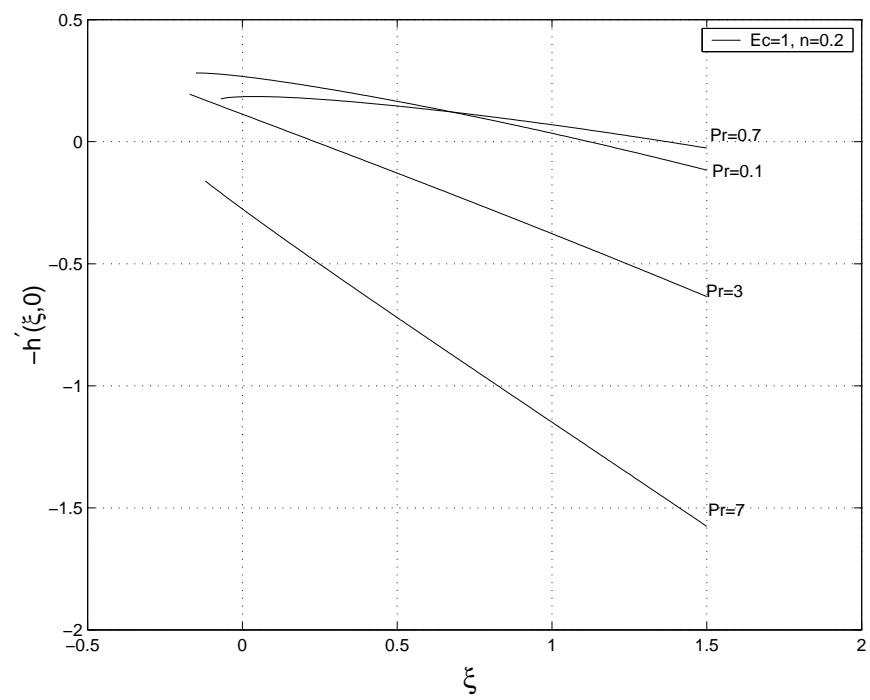

Figure 2: $-h^{\prime}(\xi, 0)$ plotted against $\xi$, for $E c=1, n=0.2$ and $\operatorname{Pr}=0.1,0.7,7$. 


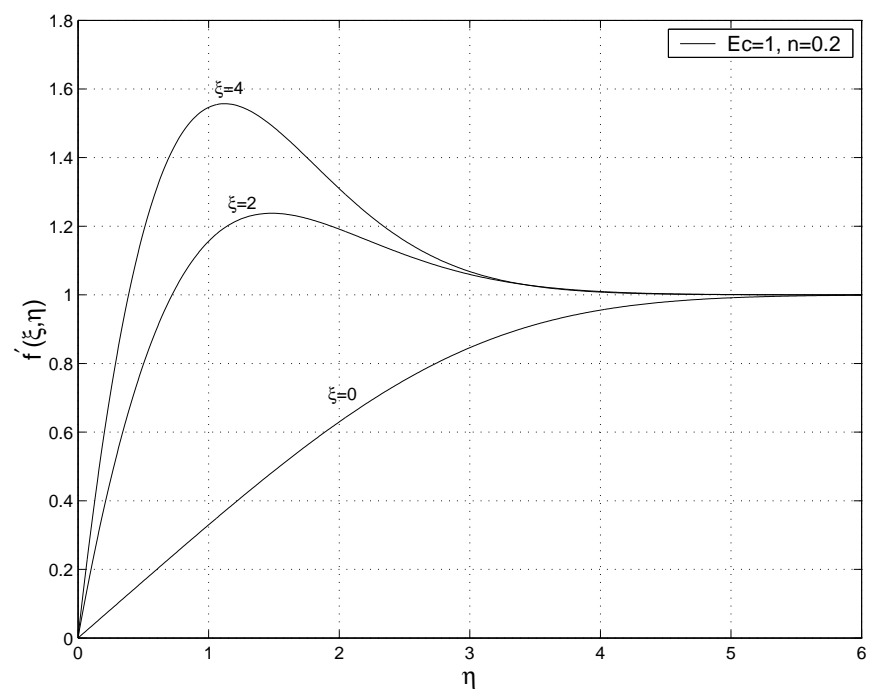

Figure 3: $f^{\prime}(\xi, \eta)$ plotted against $\eta$, for $\operatorname{Pr}=0.7, E c=1, n=0.2$ and $\xi=0,2,4$.

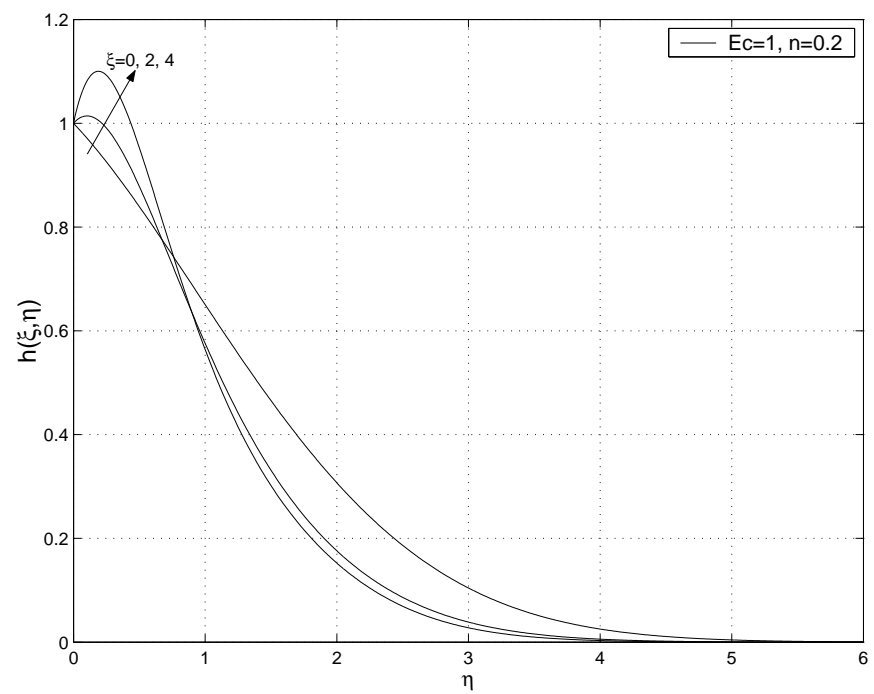

Figure 4: $h(\xi, \eta)$ plotted against $\eta$, for $\operatorname{Pr}=0.7, E c=1, n=0.2$ and $\xi=0,2,4$. 


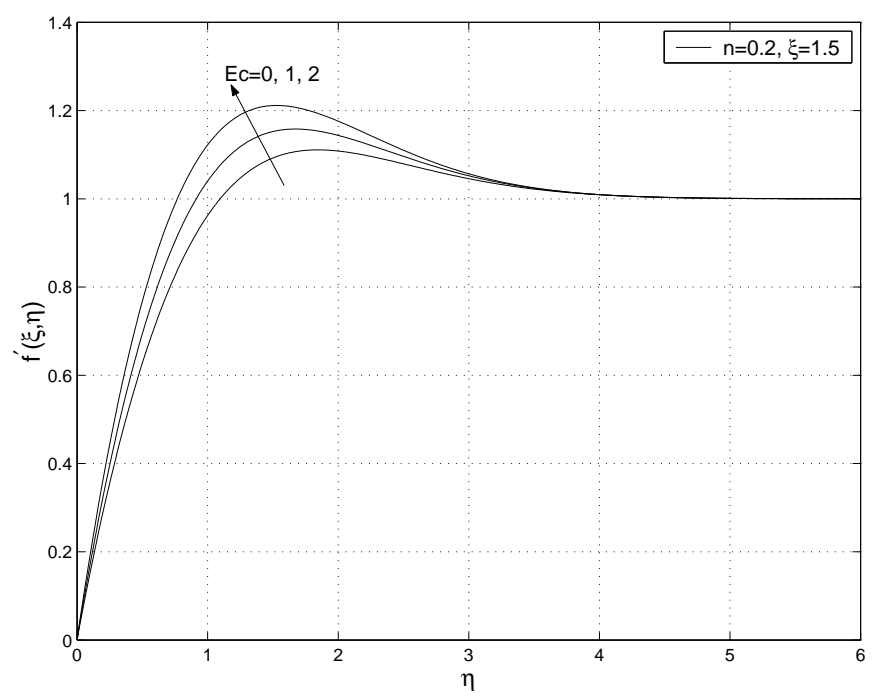

Figure 5: $f^{\prime}(\xi, \eta)$ plotted against $\eta$, for $n=0.2, \xi=1.5, \operatorname{Pr}=0.7$ and $E c=0,1,2$.

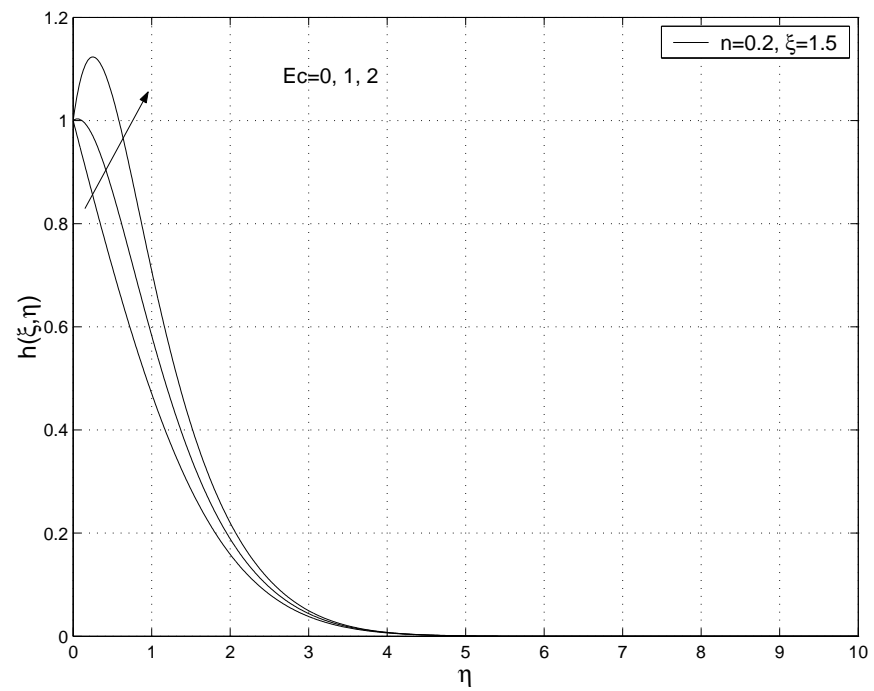

Figure 6: $h(\xi, \eta)$ plotted against $\eta$, for $n=0.2, \xi=1.5, \operatorname{Pr}=0.7$ and $E c=0,1,2$. 


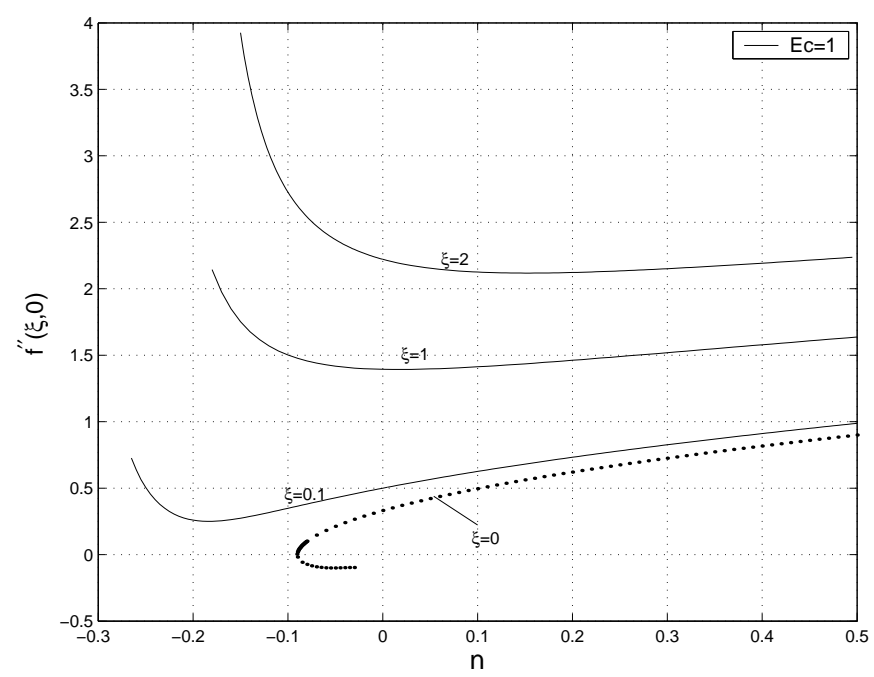

Figure 7: $f^{\prime \prime}(\xi, 0)$ plotted against $n$, for $\operatorname{Pr}=0.7, E c=1$ and $\xi=0,0.1,1,2$.

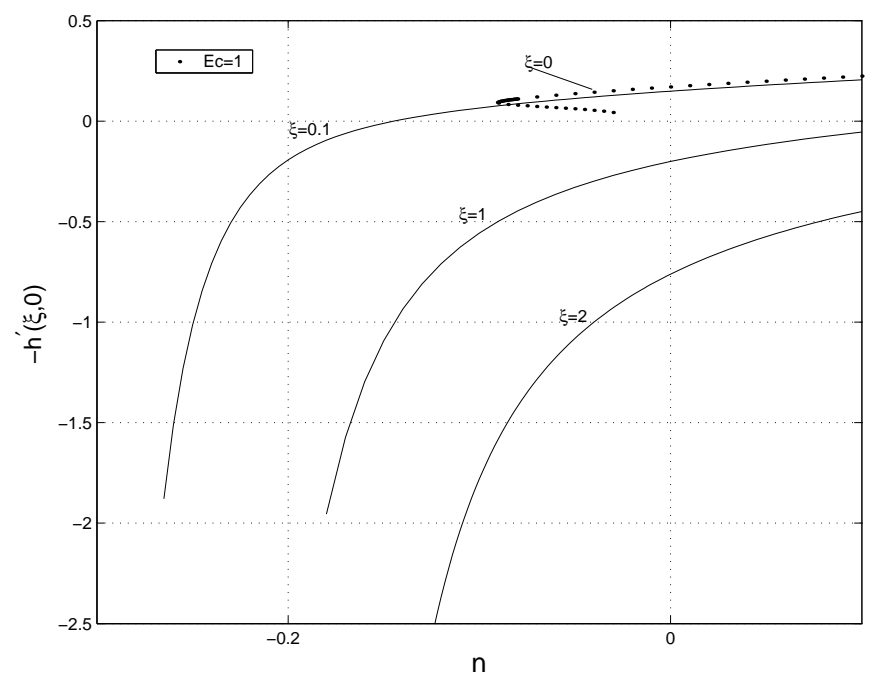

Figure 8: $-h^{\prime}(\xi, 0)$ plotted against $n$, for $\operatorname{Pr}=0.7, E c=1$ and $\xi=0,0.1,1,2$. 


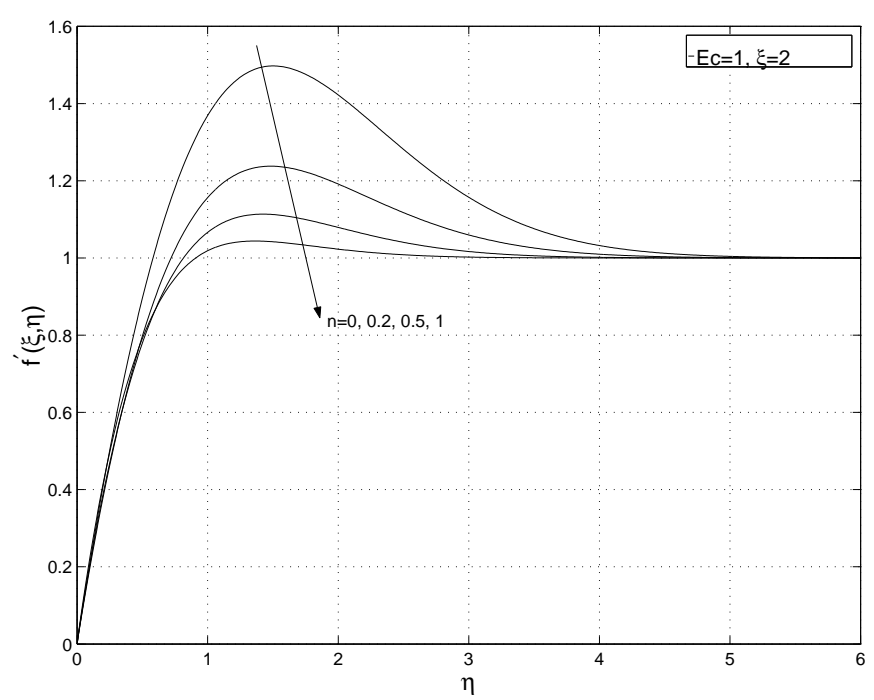

Figure 9: $f^{\prime}(\xi, \eta)$ plotted against $\eta$, for $\operatorname{Pr}=0.7, E c=1, \xi=2$ and $n=$ $0,0.2,0.5,1$.

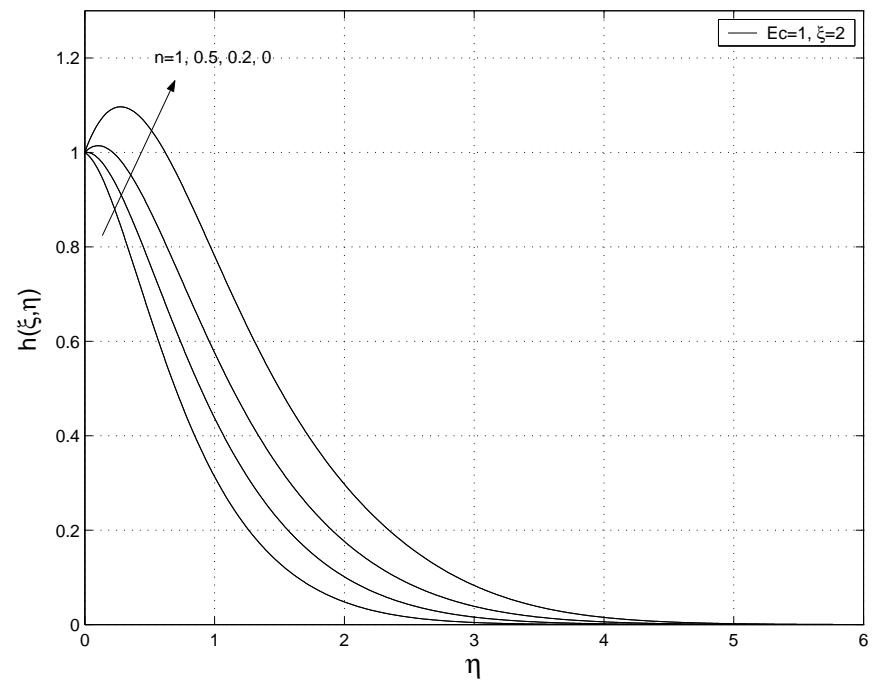

Figure 10: $h(\xi, \eta)$ plotted against $\eta$, for $\operatorname{Pr}=0.7, E c=1, \xi=2$ and $n=$ $0,0.2,0.5,1$. 


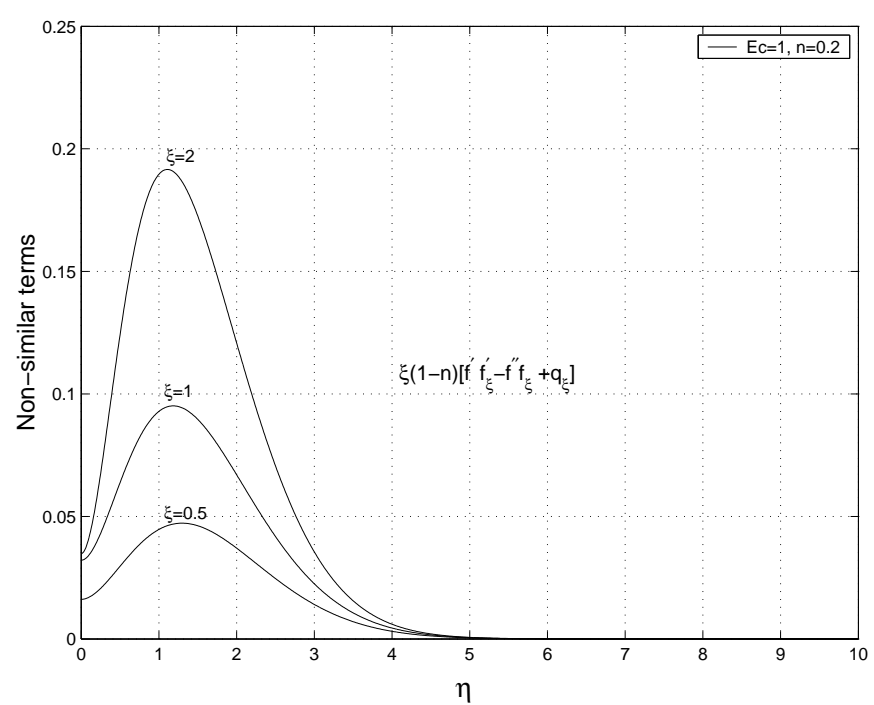

Figure 11: $(1-n) \xi\left[f^{\prime} f_{\xi}^{\prime}-f^{\prime \prime} f_{\xi}+q_{\xi}\right]$ plotted against $\eta$, for $\operatorname{Pr}=0.7, E c=1, n=0.2$ and $\xi=0.5,1,2$.

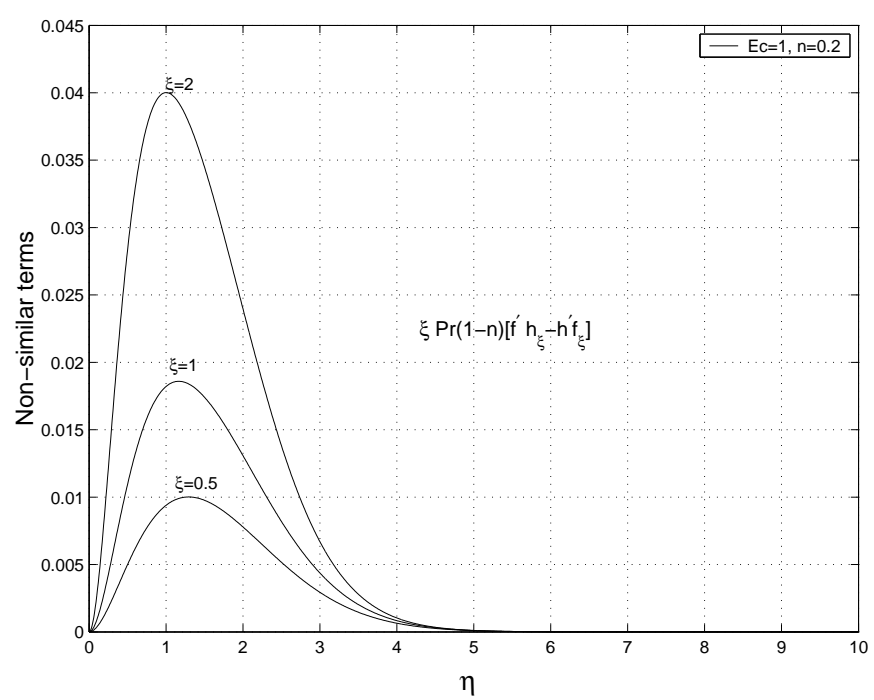

Figure 12: $(1-n) \operatorname{Pr} \xi\left[f^{\prime} h_{\xi}-h^{\prime} f_{\xi}\right]$ plotted against $\eta$, for $\operatorname{Pr}=0.7, E c=1, n=0.2$ and $\xi=0.5,1,2$. 
572 Local non-similarity solutions for a forced-free boundary-layer flow

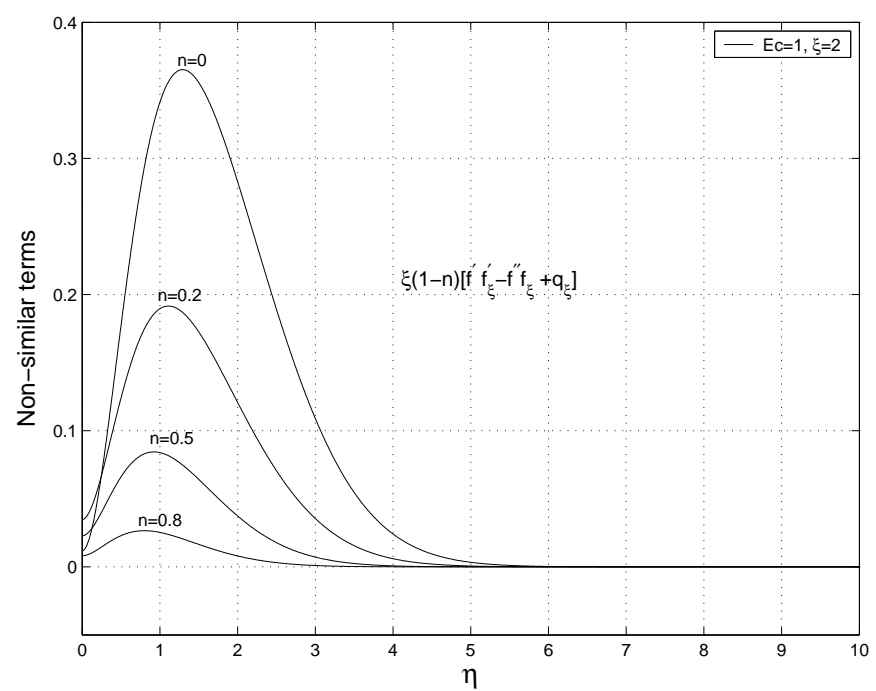

Figure 13: $(1-n) \xi\left[f^{\prime} f_{\xi}^{\prime}-f^{\prime \prime} f_{\xi}+q_{\xi}\right]$ plotted against $\eta$, for $\operatorname{Pr}=0.7, E c=1, \xi=2$ and $n=0,0.2,0.5,0.8$.

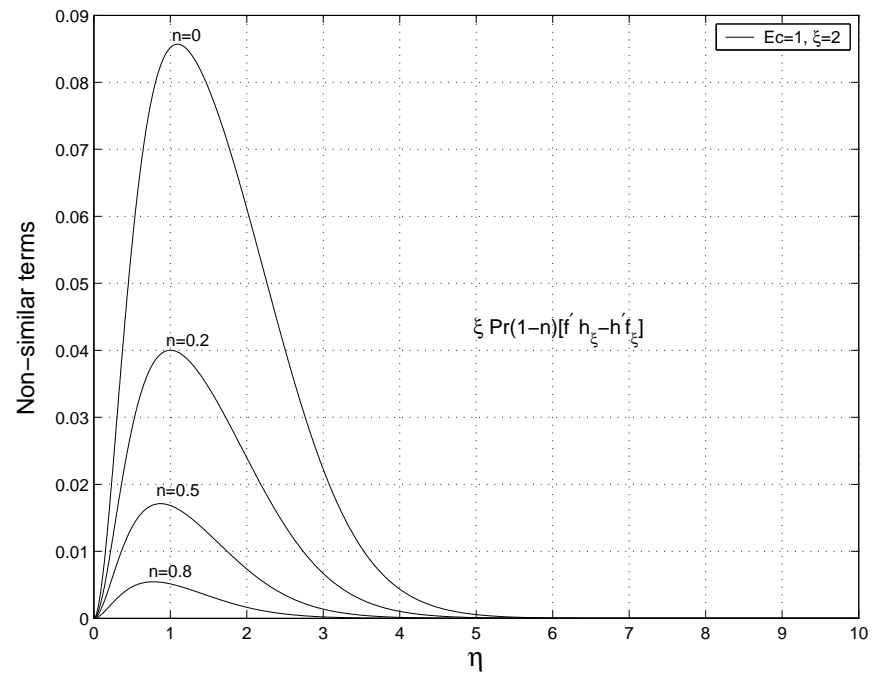

Figure 14: $(1-n) \operatorname{Pr} \xi\left[f^{\prime} h_{\xi}-h^{\prime} f_{\xi}\right]$ plotted against $\eta$, for $\operatorname{Pr}=0.7, E c=1, \xi=2$ and $n=0,0.2,0.5,0.8$. 


\section{REFERENCES}

[1] T.S. Chen, Parabolic systems: Local non-similarity method, in: W.J. Minkowycz, E.M. Sparrow, G.E. Schneider (Eds.), Handbook of numerical heat transfer, John Wiley \& Sons, New York, 183-214, 1988.

[2] I.A. Hassanien, F.S. Ibrahim, S.R.G. Rama, Mixed convection boundary layer flow of a micropolar fluid on a horizontal plate, Chem. Eng. Comm. 170, 117131, 1998.

[3] Y. Mori, Buoyancy effects in forced laminar flow over a horizontal plate. Journal of Heat Transfer, 83, 479-482, 1961.

[4] E.W. Mureithi, D.P. Mason, On the stability of a forced-free boundary layer flow with viscous heating, Fluid Dynamics Research 31, 65-78, 2002.

[5] M.S. Raju, X.Q. Liu, C.K. Law, A formulation of combined force and free convection past horizontal and vertical surfaces, Int. J. Heat Mass Transfer 27, 2215-2224, 1984.

[6] N. Ramachandran, B.F. Armaly, T.S. Chen, Mixed convection over a horizontal plate, J. Heat Transfer 105, 420-423, 1983.

[7] W.R. Risbeck, T.S. Chen, B.F. Armaly, Laminar mixed convection on horizontal flat plates with variable surface heat flux, Int. J. Heat Mass Transfer 37, 699-704, 1994.

[8] H. Schlichting, K. Gersten, Laminar boundary layer theory, Springer-Verlag, Berlin Heidelberg, 172-173, 1968.

[9] E.M. Sparrow, W.J. Minkowycz, Buoyancy effects on horizontal boundary layer flow and heat transfer, Int J. Heat Mass Transfer 5, 505-511, 1962.

[10] E.M. Sparrow, , H.S. Yu, Local non-similarity thermal boundary layer solutions, J. Heat Transfer 93, 328-334, 1971.

[11] E.M. Sparrow, H. Quack, C.J. Boerner, Local non-similarity boundary layer solutions, AIAA 8,1936-1942, 1970.

[12] D.F. Rogers, Laminar flow analysis, Cambridge University Press, 1992. 